\title{
Application of POCUS in patients with COVID-19 for acute respiratory distress syndrome management: a narrative review
}

\author{
Xuehui Gao ${ }^{1}$, Xiaojing Zou' ${ }^{1}$, Ruiting Li ${ }^{1}$, Huaqing Shu' ${ }^{1}$, Yuan Yu ${ }^{1}$, Xiaobo Yang ${ }^{1}$ and You Shang ${ }^{1,2^{*}}$
}

\begin{abstract}
COVID-19 has inflicted the world for over two years. The recent mutant virus strains pose greater challenges to disease prevention and treatment. COVID-19 can cause acute respiratory distress syndrome (ARDS) and extrapulmonary injury. Dynamic monitoring of each patient's condition is necessary to timely tailor treatments, improve prognosis and reduce mortality. Point-of-care ultrasound (POCUS) is broadly used in patients with ARDS. POCUS is recommended to be performed regularly in COVID-19 patients for respiratory failure management. In this review, we summarized the ultrasound characteristics of COVID-19 patients, mainly focusing on lung ultrasound and echocardiography. Furthermore, we also provided the experience of using POCUS to manage COVID-19-related ARDS.
\end{abstract}

Keywords: COVID-19, SARS-CoV-2, Acute respiratory distress syndrome, ARDS, Point-of-Care ultrasound, POCUS

\section{Background}

The coronavirus disease 2019 (COVID-19), caused by severe acute respiratory syndrome coronavirus 2 (SARS$\mathrm{CoV}-2)$, is highly transmittable and potentially lethal $[1$, 2]. Although most patients with COVID-19 have mild manifestations, approximately $15 \%$ to $30 \%$ of hospitalized patients deteriorate, requiring admission to an intensive care unit (ICU) or invasive mechanical ventilation for acute respiratory distress syndrome (ARDS) [3], which seriously affects their prognosis $[4,5]$. However, some experts suggested that the COVID-19-related ARDS was somewhat different from classical ARDS, because of a relatively preserved compliance of the respiratory system despite marked hypoxemia [6-8]. Furthermore, the highly contagious nature of SARS-CoV-2 also increases management difficulties. Point-of-care ultrasound (POCUS) - a convenient bedside tool for

\footnotetext{
*Correspondence: you_shanghust@163.com

${ }^{1}$ Department of Critical Care Medicine, Union Hospital, Tongji Medical College, Huazhong University of Science and Technology, No.1277, Jiefang Avenue, Wuhan 430022, Hubei, People's Republic of China Full list of author information is available at the end of the article
}

monitoring pulmonary function and assessing related complications-is considered as a valid aid in COVID-19 patient management [9]. At present, there are few summaries on POCUS guiding the management of COVID19-related ARDS. In this review, we will summarize the ultrasonographic characteristics of COVID-19 patients, mainly focusing on the lung ultrasound and echocardiography. Furthermore, we also provided the experience of using POCUS to manage COVID-19-related ARDS (Table 1).

\section{Main text \\ POCUS in COVID-19-related ARDS}

Ultrasonography, a safe and effective form of imaging, has been used by physicians to aid in diagnosis and guide procedures for more than half a century. Over the past two decades, ultrasound equipment has become more compact, higher functional, and less expensive, which has facilitated the growth of POCUS, an ultrasound performed and interpreted by the clinician at the bedside. It is this feature that lead to the broad applications of 
Table 1 Summarizing the different uses of the different POCUS modalities in COVID-19 related ARDS

\begin{tabular}{ll}
\hline POCUS modalities & The different uses of the different POCUS modalities \\
\hline Lung ultrasound & 1. Allowing to rule in or rule out COVID-19 pneumonia combined with a medical history during the pandemic [17] \\
& 2. Screening the patient and aiding precise triage and treatment allocation in both the emergency and ICU departments \\
& 3. Assessing the lung lesions' progress and evaluating the severity of COVID-19 [10, 12, 18, 19] \\
& 4. Combing with echocardiography, diaphragmatic ultrasound and other aspects to guide the comprehensive treat- \\
& ments for COVID-19 related ARDS and monitor the response to treatments \\
1. Identifying serious RV complications such as ACP, pulmonary embolism, pulmonary hypertension, etc. [29-33, 73] & 2. Detecting other complications related to LV dysfunction [30] \\
3. Guiding the comprehensive treatments for COVID-19 related ARDS, mainly the management of cardiac complications \\
and hemodynamics \\
1. IVC ultrasound combined with LUS and echocardiography is used for fluid resuscitation administration [79] \\
2. Thoracic and abdominal ultrasounds help detect free fluid, empyema, pneumothorax, or cardiac tamponade \\
3. Vascular ultrasound helps identify DVT and guide catheterization \\
4. Obstetric ultrasound helps manage pregnant women infected with COVID-19 [39]
\end{tabular}

POCUS in the ongoing battle against the COVID-19 pandemic [10-12].

\section{Lung ultrasound}

The SARS-CoV-2 infection mainly affects the respiratory system. Although chest CTs are the gold standard for lung imaging, a systematic CT scan during the pandemic is not feasible for many reasons, such as the risk of SARS-CoV-2 transmission when infected patients are transferred to radiology facilities. Thus, Lung ultrasound (LUS) was validated for lung aeration monitoring in ARDS patients with a good correlation with chest CTs [13]. A retrospective comparison study demonstrated that LUS is more sensitive than chest CT to detect lesions in COVID-19 patients [14].

LUS can rapidly define alterations affecting the ratio between tissue and air in the superficial lung by trained clinicians. COVID-19 pneumonia imaging typically shows bilateral multilobar ground-glass opacities or consolidative opacities with a peripheral, or posterior, distribution. These characteristics provide an ideal fundamentals for LUS $[15,16]$. Recent multi-center studies showed that in patients suspected for COVID-19, LUS patterns of probability combined with a medical history allowed to rule in or rule out COVID-19 pneumonia at bedside with high accuracy [17]. In the early and mild infection stage, focal B lines are the main pattern; in the progressive stage and critically ill patients, thickened pleural line, multiple, or fused B-lines and subpleural non-homogeneous consolidations are the main features; in an advanced stage, the main features are multiple continuous large-scale hypoechoic consolidation with hepatization sign, diffused B-lines, and extensive air bronchogram sign $[10,12,18]$. Furthermore, pleural line thickening with uneven $B$ lines is a notable pattern, suggesting that the patient may be complicated by pulmonary fibrosis, an important factor influencing pulmonary function in COVID-19 patients [18]. Additionally, recent studies proposed a more precise evaluation standard, namely LUS score (LUSS), and demonstrated that LUSS was correlated to disease severity and progression [19], and had a good agreement with COVID-19 patients outcomes [20-22].

LUS also has some limitations in COVID-19 and there are occasions in which chest CTs are needed. First, it should be highlighted that all lesions must be extended to the pleural surface to be detected by LUS, because examination of the central structures is prevented by the barrier created by the pleural-lung interface [23]. And consolidation has to be within an intercostal window. This means that a small number of cases will not be identified by LUS. Moreover, LUS is often unable to identify lesion chronicity, limiting its utility in a person with pre-existing pulmonary conditions [11]. Additionally, although the sensitivity of LUS is high, its specificity may not be the same. Especially in the post-epidemic era, it is difficult to distinguish CVOID-19 from other causes of pneumonia (atypical bacteria, flu viruses, etc.) [24].

\section{Echocardiography}

About $20 \%$ of hospitalized patients and up to $67 \%$ of critically ill patients with COVID-19 will develop ARDS [5, 25]. Cardiac failure, in particular right ventricular (RV) dysfunction, is commonly encountered in moderate to severe ARDS. In the early stages of the pandemic, some reports have indicated that cardiac complications not only were common in COVID-19 infection but also are associated with increased mortality [26, 27]. The high mortality and yet unknown aspects of the COVID-19 clinical course necessitate cardiac evaluations. 
The most frequent cardiac abnormality is RV dysfunction among patients with COVID-19 infection [28]. RV dysfunction by echocardiography is commonly defined as the presence of pressure and/or volume overload of the right ventricle [29]. RV volume overload is defined as RV dilation and RV pressure overload is defined as dyskinetic movement of the septum during end-systole. According to the American Society of Echocardiography RV dysfunction is present when the parameters to quantify RV function are less than the lower value of the normal range: tricuspid annular plane systolic excursion is less than $17 \mathrm{~mm}$, pulsed Doppler S wave is less than $9.5 \mathrm{~cm}$ per second, RV fractional area change is less than $35 \%$, RV ejection fraction is less than $45 \%$ [30]. In the RV-focused view, RV diameter greater than $41 \mathrm{~mm}$ at the base and greater than $35 \mathrm{~mm}$ at midlevel indicates chamber dilatation [30]. Clinically, a simpler and more intuitive evaluation method is to compare the right ventricular end diastolic area (RVEDA) with the left ventricular end diastolic area (LVEDA) [31, 32]. Right ventricular dilation is defined as moderate when RVEDA/LVEDA ratio is between 0.6 and 1 , and as severe when this ratio is more than 1.0 [33]. Additionally, RV dilatation can cause shifting of the interventricular septum toward the left, the " $\mathrm{D}$ sign" also is a simple method to assess RV size.

Due to 'ventricular interdependence', RV dilatation can impede left ventricular diastolic filling, resulting in left ventricular (LV) dysfunction. Recently, A large study about Doppler echocardiography indicated that LV haemodynamics can aid in risk stratification of patients with severe COVID-19, and LV low output and elevated filling pressure are independent predictors of mortality [34]. Two-dimensional and three-dimensional echocardiography can realize real-time measurement of LV diameters, volumes, ejection fraction, and mass, and even finer measurement, including the peak early filling (E wave) and late diastolic filling (A wave) velocities, E/A ratio, lateral annular velocities $\left(\mathrm{e}^{\prime}\right)$, and deceleration time of early filling velocity [30]. García-Cruz et al. proposed an algorithm, the ORACLE protocol, to perform a quick systemic evaluation of biventricular function, valvular heart disease, pericardial effusion, hemodynamics, and lung ultrasound using POCUS [35].

\section{Other aspects}

Many critically ill COVID-19 patients develop secondary muti-organ dysfunction [36], including acute kidney injury (AKI), liver injury gastrointestinal disturbance, cardiac dysfunction, as well as hypercoagulability with thromboembolic events, such as ischemic stroke. These dysfunctions may be evaluated with POCUS. POCUS can easily identify post-renal and pre-renal causes of AKI by evaluating hemodynamics assessment and post-renal obstrucations. Also, it can assess acute gastrointestinal complications in COVID-19 patients, including cholestasis and bowel ischemia [37], and detect circulating microemboli within cerebral arteries using transcranial Doppler ultrasound [38]. In addition, ultrasonography has been used in obstetrics for decades, with no evidence of harmful effects at normal diagnostic levels. POCUS facilitate care for pregnant women with SARS-CoV-2 infection and their babies [39].

Treatment for COVID-19-related ARDS Patients with POCUS POCUS is vital in COVID-19-related ARDS Patients because it provides real time information for diagnosis and treatment. Figure 1 presents a road map for the possible comprehensive use of POCUS in COVID-19 ARDS.

\section{Ventilation support and open-lung}

The mainstream of treatment for COVID-19 patients is still supportive care. Ventilation support remains the most important aspect. However, ventilation strategies vary for patients at different stages of the disease. POCUS can be used to track the course of COVID-19 pneumonia and monitor the response to ventilation support.

High-flow nasal oxygen and non-invasive ventilation are reserved for patients in the early stage of ARDS [40]. However, considering rapid clinical deterioration in severe COVID-19 patients and self-inflicted lung injury caused by respiratory distress, delayed intubation may lead to emergent intubations and mortality. POCUS is a valuable tool for close and real-time monitoring of lung function, which can be used to determine the timing of invasive mechanical ventilation, reducing the exposure risk of health-care workers in emergencies. A recent study showed that a LUSS $\geq 25$ on admission had $90 \%$ specificity for intubation, and could be a warning for exacerbations in critically ill COVID-19 patients [20]. For intubated COVID-19 patients, instead of auscultation, LUS examination can confirm endotracheal intubation by examining lung sliding of both side.

In the early stage of mechanical ventilation in COVID19, liberal tidal volume and relatively low PEEP levels were recommended [7]. With the disease progress, the protective ventilation strategy with higher PEEP levels may be beneficial. However, inappropriate high PEEP levels will decrease pulmonary compliance and impact right heart function. Performing POCUS to monitor the size of both ventricles and contractility while monitoring lung function, helps to tailor the ventilatory strategy, reaching a balance between lung opening and adequate right heart function [41, 42].

If hypoxaemia progresses to a $\mathrm{P} / \mathrm{F}$ ratio lower than $150 \mathrm{mmHg}$, PEEP, recruitment maneuvers (RM) and prone position should be considered [42-44]. Lung 


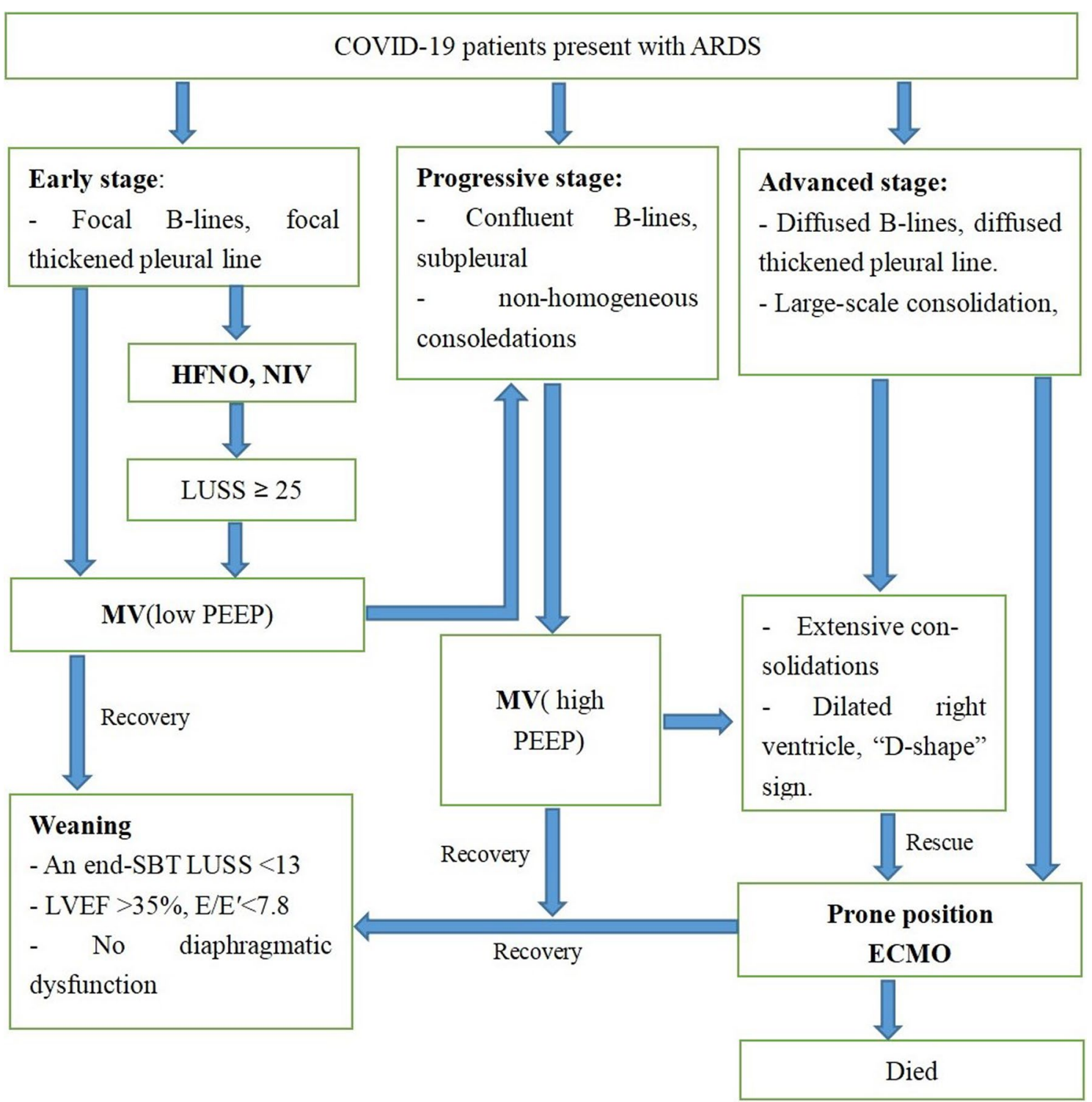

Fig. 1 A road map for the possible comprehensive use of POCUS in COVID-19 ARDS. MV mechanical ventilation, PEEP positive end expiratory pressure, SBT spontaneous breathing trial, LUS lung ultrasound, LVEF left ventricular ejection fraction, E pulsed wave doppler early mitral valve inflow velocity, E' mitral annular tissue doppler velocity, ECMO extracorporeal membrane oxygenation

RM plus PEEP increases airway pressure to open collapsed alveoli and keep them open throughout the ventilatory cycle. When PEEP is increased stepwisely, lung recruitment regional analysis of independent and nondependent regions may assist the early recognition of responders. Prone position ventilation, that, has the potential to attenuate ventilator-related lung injury and unload the right ventricle while correcting hypoxemia and hypercapnia, should be applied early $[45,46]$. At least $12-16 \mathrm{~h}$ per day of prone position is strongly recommended for patients with severe ARDS [47, 48]. During these prolonged sessions without transthoracic windows, transesophageal echocardiography is a helpful and necessary tool when these patients undergo persistent respiratory compromise or hemodynamic deterioration with no obvious explanations. Lung ventilation can also be monitored in the lateral and posterior regions of the thorax.

\section{Extracorporeal membrane oxygenation}

Extracorporeal membrane oxygenation (ECMO) is initiated in cases of refractory hypoxemia [49]. V-V ECMO is the primary mode used in COVID-19 patients [44]. 
However, some patients with COVID-19 have respiratory failure integrated with cardiovascular system damage and circulatory failure, and V-A ECMO is used in this situation. Echocardiographic examination is necessary before catheterization, because V-A ECMO instead of V-V ECMO may be a better choice, when severe left heart dysfunction presents [50]. During catheterization, vascular ultrasound enhances the safety and efficacy with real-time guidance. Improper placement of the cathetermay increase recirculation and reduce the oxygenation efficiency of ECMO. Therefore, ultrasound should be performed after peripheral cannulation to confirm the correct position of the catheter [51]. Additionally, POCUS also plays an important role in the daily management of ECMO patients. POCUS is a valid cardiac output monitoring complement of thermodilution and pulse contour analysis $[52,53]$.

\section{Weaning and extubation}

For patients gradually recovering respiratory function, earlier liberation from invasive mechanical ventilation reduces the incidence of ventilator-associated complications. However, the risks of premature extubation and subsequent reintubation cannot be avoided completely, which would cause additional harm to patients and increase the risk of viral exposure in healthcare workers. One argument surrounding the challenges of primary extubation shows that COVID-19 patients had a higher chance of re-intubation than non-COVID-19 patients [54]. Evaluation of weaning indications in patients undergoing invasive mechanical ventilation remains a great challenge. POCUS functions as a comprehensive method to predict failed extubation through cardiac, lung, and diaphragmatic dysfunction parameters [55]. There is a positive correlation between echocardiographic measurements of diastolic dysfunction, particularly $E / E^{\prime}$, and weaning failure [56, 57]. A study suggested that patients are more likely to failed weaning when the left ventricular ejection fraction is lower than $35 \%$ and $E / E^{\prime}$ is greater than 7.8 [56]. LUSS may be a simple tool to accurately predict post-extubation distress based on a spontaneous breathing trial (SBT) $[58,59]$. An end-SBT LUSS $<13$ predicted extubation success, and an end-SBT LUSS $>17$ predicted postextubation distress [58]. Active diaphragm contraction may increase aeration of posterior and dependent lung regions, promoting extubation success. Diaphragmatic function indices, including diaphragmatic excursion and thickening fraction, can also be rapidly obtained using POCUS [55].

Percutaneous tracheostomy is a common procedure of modern critical care. It benefits patients who require prolonged ventilation, allowing for a more controlled wean. Considering that patients with COVID-19 typically have longer periods of ventilation than patients with other viral pneumonias [60], the use of tracheostomy to aid weaning from ventilatory support should be considered [61], which may ease the burden upon critical care resources [62]. Angel et al. showed that early tracheostomy in COVID-19 patients can be performed with improved patient prognosis [63]. Reported rates of tracheostomies during the coronavirus pandemic were significantly higher than those of pre-pandemic [61, 64, $65]$. Ultrasound scan facilitates clinicians to identify thyroid glands, and vessels anterior to trachea, to delineate the airway, to evaluate the thickness of the skin over the neck and even to visualize the needle and guides wire passage, thereby reducing the occurrence of fatal bleeding, pneumothorax, tracheoesophageal fistula and other complications [66]. In addition, ultrasound enables bedside assessment some tracheostomy-associated complications. A study reported that ultrasonography was the first choice for diagnosis of the post-tracheostomy pneumothorax [67].

\section{Management of severe complications}

The most common echocardiographic abnormality among patients with COVID-19 infection was RV dilatation and dysfunction [28], suggesting the possibility of acute cor pulmonale (ACP). ACP is a severe complication defined by RV acute dilatation with paradoxical septal motion during the end-systole in the context of acute lung disease, including ARDS and associated pulmonary vascular dysfunction [68]. The prevalence of ACP ranges from 20 to $25 \%$ in the protective mechanical ventilation era, and severe ACP is associated with a poor outcome [40]. In a large prospective observational study, Mekontso Desapp and colleagues reported that the mortality did not differ between patients with or without ACP, but that in patients with severe ACP, it was an independent predictor of mortality [69]. This could be explained by the fact that patients with mildly dilated RV may have preserved RV systolic function [69]. Real time echocardiography is useful to promptly detect mild ACP and guide interventions, including ventilatory strategy adjustment, prone positioning initiation and nitric oxide inhalation to avoid further deterioration of right ventricular function [41, 70].

It is worth noting that acute pulmonary embolism (APE) is also a potential cause of ACP in COVID-19 patients $[32,71]$. In the early stage of outbreak, elevated levels of D-dimer in COVID-19 patients attracted attentions [25]. In a study involving 58 COVID-19 patients, $14.8 \%$ had DVT despite accepting low-molecular-weight heparin prophylactic treatment [72]. When dilated right ventricule is detected, APE should be considered, and venous ultrasound should be performed, especially in 
ARDS patients who have sudden hemodynamic instability and severe hypoxemia. But we should be vigilant that negative DVT does not necessarily rule out APE. Patients with APE often have pulmonary hypertension. The pulmonary artery systolic pressure is most commonly obtained by a modified Bernoulli equation to convert peak tricuspid regurgitation velocity into pressure and to add the right atrial pressure. Additionally, in the absence of reliable tricuspid regurgitant signals, the pulmonary ejection signal acceleration time (PACT) also can be used to assess pulmonary artery pressure. For example, a PACT of 70-90 ms indicates a pulmonary artery systolic pressure $>70 \mathrm{mmHg}[73]$.

\section{Hemodynamics and fluid administration}

Significant hemodynamic derangement can accompany COVID-19-related ARDS. In a large cohort of COVID19 patients, the incidence of circulatory shock was 30\% [74]. Identifying the etiology and mechanism of shock is important to select the best therapy. These patients are initially resuscitated with fluid, and POCUS should be performed if hemodynamic instability persists.

Fluid resuscitation is essential in patients with shock. For example, severe hemorrhagic shock can lead to outflow tract obstruction if the patient is under-resuscitated, especially for patients with the LV exhibits significant asymmetric septal, or severe concentric, hypertrophy. However, some studies reported that positive fluid balance is an independent risk factor for ARDS development [75]. RV failure can be precipitated if excessive fluid resuscitation is undertaken which, in turn, may cause shifting of the interventricular septum toward the left impeding LV diastolic filling [76]. This would lead to pulmonary edema and hypoxemia which will further worsen RV failure, thus forming a vicious circle. The optimal fluid management for ARDS patients is key to restore adequate organ perfusion while avoiding diffuse tissue edema and positive fluid balance [77]. LUS is sensitive to pulmonary edema with the pattern of "comet tails", which functions as a "safeguard" against excessive fluid by dynamic evaluation. It can be used along with simple cardiac and vena cava analysis for fluid resuscitation administration [78].

Inferior vena cava (IVC) ultrasound examination should be performed as the first step to identify the etiology of shock [79]. However, there are limitations to use the IVC alone to estimate mean systemic venous pressure. In order to identify the etiology of shock, it is also necessary to examine the heart. Doppler examination of the hepatic venous flow can also be added [80]. In patients with reduced/increased mean systemic venous pressure, further ultrasound examination of the thoracic and abdominal should be considered to detect free fluid, empyema, pneumothorax, or cardiac tamponade. Subsequent treatment can be tailored according to the etiology of shock.

\section{Conclusion}

SARS-CoV-2 can cause bilateral pneumonia. Among those with pneumonia, 20-42\% have developed ARDS [81], which seriously affects COVID-19 prognosis. POCUS is a rapid, feasible, and safe bedside tool for respiratory and hemodynamic evaluation of patients with COVID-19. This review provides information about the use of POCUS in COVID-19 patients with ARDS. It might help intensivists optimize and make individualized management for these patients.

\section{Abbreviations \\ COVID-19: Coronavirus disease 2019; SARS-CoV-2: Severe acute respiratory syndrome coronavirus 2; WHO: World Health Organization; ARDS: Acute respiratory distress syndrome; POCUS: Point-of-care ultrasound; ICU: Intensive care unit; CT: Computed tomography; PEEP: Positive end expiratory pressure; ECMO: Extracorporeal membrane oxygenation; LUS: Lung ultrasound; RM: Recruitment maneuvers; LUSS: Lung ultrasound score; SBT: Spontaneous breathing trial; ACP: Acute cor pulmonale; RVEDA: The right ventricular end-diastolic area; LVEDA: The left ventricular end-diastolic area; APE: Acute pulmonary embolism; DVT: Deep vein thrombosis; RV: Right ventricular; LV: Left ventricular; AKI: Acute kidney injury; IVC: Inferior vena cava.}

\section{Acknowledgements}

Not applicable.

\section{Authors' contributions}

XHG and YS contributed substantially to the article concept. XHG, XJZ and RTL retrieved literature and manuscript writing. HQS, YY, XBY and $Y S$ revised the manuscript. YS reviewd and approved the final version before submission. All the listed authors have participated actively in the study. All authors read and approved the final manuscript.

Funding

No funding.

Availability of data and materials

Not applicable.

\section{Declarations}

Ethics approval and consent to participate Not applicable.

Consent for publication

Not applicable.

Competing interests

The authors declare that they have no competing interests.

\section{Author details}

${ }^{1}$ Department of Critical Care Medicine, Union Hospital, Tongji Medical College, Huazhong University of Science and Technology, No.1277, Jiefang Avenue, Wuhan 430022, Hubei, People's Republic of China. Institute of Anesthesiology and Critical Care Medicine, Union Hospital, Tongji Medical College, Huazhong University of Science and Technology, Wuhan 430022, Hubei, People's Republic of China. 
Received: 17 September 2021 Accepted: 24 January 2022

Published online: 05 February 2022

\section{References}

1. Zhu N, Zhang D, Wang W, et al. China novel coronavirus investigating and research team. A novel coronavirus from patients with pneumonia in China, 2019. N Engl J Med. 2020;382(8):727-33.

2. Wu F, Zhao S, Yu B, et al. A new coronavirus associated with human respiratory disease in China. Nature. 2020;579(7798):265-9.

3. Chen N, Zhou M, Dong X, et al. Epidemiological and clinical characteristics of 99 cases of 2019 novel coronavirus pneumonia in Wuhan, China: a descriptive study. Lancet. 2020;395(10223):507-13.

4. Arentz M, Yim E, Klaff L, et al. Characteristics and outcomes of 21 critically III patients with COVID-19 in Washington State. JAMA. 2020;323(16):1612-4.

5. Yang $X, Y u Y, X u$ J, et al. Clinical course and outcomes of critically ill patients with SARS-CoV-2 pneumonia in Wuhan, China: a singlecentered, retrospective, observational study. Lancet Respir Med. 2020;8(5):475-81.

6. Gattinoni L, Coppola S, Cressoni M, et al. COVID-19 does not lead to a "typical" acute respiratory distress syndrome. Am J Respir Crit Care Med. 2020:201(10):1299-300.

7. Gattinoni L, Chiumello D, Caironi P, et al. COVID-19 pneumonia: different respiratory treatments for different phenotypes? Intensive Care Med. 2020;46(6):1099-102.

8. Guarracino F, Vetrugno L, Forfori F, et al. Lung, heart, vascular, and diaphragm ultrasound examination of COVID-19 patients: a comprehensive approach. J Cardiothorac Vasc Anesth. 2021;35(6):1866-74.

9. Moore CL, Copel JA. Point-of-care ultrasonography. N Engl J Med. 2011;364(8):749-57.

10. Soldati $G$, Smargiassi $A$, Inchingolo $R$, et al. Is there a role for lung ultrasound during the COVID-19 pandemic? J Ultrasound Med. 2020:39(7):1459-62.

11. Cheung JC, Lam KN. POCUS in COVID-19: pearls and pitfalls. Lancet Respir Med. 2020;8(5):e34.

12. Chinese Expert Consensus on Critical Care Ultrasound Applications at COVID-19 Pandemic (2020). Department of Radiology Faculty Papers. Paper 84. https://jdc.jefferson.edu/radiologyfp/84.

13. Chiumello D, Mongodi S, Algieri I, et al. Assessment of lung aeration and recruitment by CT scan and ultrasound in acute respiratory distress syndrome patients. Crit Care Med. 2018;46(11):1761-8.

14. Yang $Y$, Huang $Y$, Gao $F$, et al. Lung ultrasonography versus chest $C T$ in COVID-19 pneumonia: a two-centered retrospective comparison study from China. Intensive Care Med. 2020;46(9):1761-3.

15. Salehi S, Abedi A, Balakrishnan S, et al. Coronavirus disease 2019 (COVID19): a systematic review of imaging findings in 919 patients. AJR Am J Roentgenol. 2020;215(1):87-93.

16. Ashton-Cleary DT. Is thoracic ultrasound a viable alternative to conventional imaging in the critical care setting? $\mathrm{Br} J$ Anaesth. 2013;111(2):152-60.

17. Volpicelli G, Gargani L, Perlini S, et al. On behalf of the International Multicenter Study Group on LUS in COVID-19. Lung ultrasound for the early diagnosis of COVID-19 pneumonia: an international multicenter study. Intensive Care Med. 2021;47(4):444-54.

18. Peng QY, Wang XT, Zhang LN. Chinese critical care ultrasound study group (CCUSG). Findings of lung ultrasonography of novel corona virus pneumonia during the 2019-2020 epidemic. Intensive Care Med. 2020;46(5):849-50.

19. Dargent A, Chatelain E, Si-Mohamed S, et al. COVIDLUS study group. Lung ultrasound score as a tool to monitor disease progression and detect ventilator-associated pneumonia during COVID-19-associated ARDS. Heart Lung. 2021;50(5):700-5.

20. de Alencar JCG, Marchini JFM, Marino LO, et al. COVID U. S. P. Registry Team. Lung ultrasound score predicts outcomes in COVID-19 patients admitted to the emergency department. Ann Intensive Care. 2021;11(1):6.

21. Lichter Y, Topilsky Y, Taieb P, et al. Lung ultrasound predicts clinical course and outcomes in COVID-19 patients. Intensive Care Med. 2020;46(10):1873-83
22. Brahier T, Meuwly JY, Pantet $\mathrm{O}$, et al. Lung ultrasonography for risk stratification in patients with COVID-19: a prospective observational cohort study. Clin Infect Dis. 2020; ciaa1408.

23. Soldati G, Smargiassi A, Inchingolo R, et al. Lung ultrasonography may provide an indirect estimation of lung porosity and airspace geometry. Respiration. 2014;88(6):458-68.

24. Vetrugno L, Bove T, Orso D, et al. Lung ultrasound and the COVID-19 "pattern": Not all that glitters today is gold tomorrow. J Ultrasound Med. 2020;39(11):2281-2

25. Huang C, Wang Y, Li X, et al. Clinical features of patients infected with 2019 novel coronavirus in Wuhan, China. Lancet. 2020;395(10223):497-506.

26. Zhou F, Yu T, Du R, et al. Clinical course and risk factors for mortality of adult inpatients with COVID-19 in Wuhan, China: a retrospective cohort study. Lancet. 2020;395:1054-62.

27. Guo T, Fan Y, Chen M, et al. Cardiovascular implications of fatal outcomes of patients with coronavirus disease 2019 (COVID-19). JAMA Cardiol. 2020:5(7):811-8.

28. Szekely Y, Lichter $Y$, Taieb $P$, et al. Spectrum of cardiac manifestations in COVID-19: a systematic echocardiographic study. Circulation. 2020;142(4):342-53.

29. Jardin F, Dubourg O, Bourdarias JP. Echocardiographic pattern of acute cor pulmonale. Chest. 1997;111(1):209-17.

30. Lang RM, Badano LP, Mor-Avi V, et al. Recommendations for cardiac chamber quantification by echocardiography in adults: an update from the American Society of Echocardiography and the European Association of Cardiovascular Imaging. J Am Soc Echocardiogr. 2015;28(1):1-39.

31. Frémont $B$, Pacouret $G$, Jacobi $D$, et al. Prognostic value of echocardiographic right/left ventricular end-diastolic diameter ratio in patients with acute pulmonary embolism: results from a monocenter registry of 1416 patients. Chest. 2008;133(2):358-62.

32. Li Y, Li H, Zhu S, et al. Prognostic value of right ventricular longitudinal strain in patients with COVID-19. JACC Cardiovasc Imaging. 2020;13(11):2287-99.

33. Rudski LG, Lai WW, Afilalo J, et al. Guidelines for the echocardiographic assessment of the right heart in adults: a report from the American Society of Echocardiography endorsed by the European Association of Echocardiography, a registered branch of the European Society of Cardiology, and the Canadian Society of Echocardiography. J Am Soc Echocardiogr. 2010;23(7):685-788

34. Taieb P, Szekely Y, Lupu L, et al. Risk prediction in patients with COVID-19 based on haemodynamic assessment of left and right ventricular function. Eur Heart J Cardiovasc Imaging. 2021;22(11):1241-54.

35. García-Cruz E, Manzur-Sandoval D, Rascón-Sabido R, et al. Critical care ultrasonography during COVID-19 pandemic: the ORACLE protocol. Echocardiography. 2020;37(9):1353-61.

36. Guan WJ, Ni ZY, Hu Y, et al. China medical treatment expert group for Covid-19. Clinical Characteristics of Coronavirus Disease 2019 in China. N Engl J Med. 2020;382(18):1708-20.

37. Bhayana R, Som A, Li MD, et al. Abdominal imaging findings in COVID-19: preliminary observations. Radiology. 2020;297(1):E207-15.

38. Tsivgoulis G, Alexandrov AV, Sloan MA. Advances in transcranial Doppler ultrasonography. Curr Neurol Neurosci Rep. 2009;9(1):46-54.

39. Driul L, Meroi F, Cecchini F, et al. COVID-19 pandemic in an Italian obstetric department: sharing our experience. Acta Biomed. 2021;92(3):e2021217

40. Rochwerg B, Granton D, Wang DX, et al. High flow nasal cannula compared with conventional oxygen therapy for acute hypoxemic respiratory failure: a systematic review and meta-analysis. Intensive Care Med. 2019:45(5):563-72.

41. Mekontso Dessap A, Boissier F, Charron C, et al. Acute cor pulmonale during protective ventilation for acute respiratory distress syndrome: prevalence, predictors, and clinical impact. Intensive Care Med. 2016:42(5):862-70.

42. Bein T, Grasso S, Moerer O, et al. The standard of care of patients with ARDS: ventilatory settings and rescue therapies for refractory hypoxemia. Intensive Care Med. 2016;42(5):699-711.

43. Guérin C, Reignier J, Richard JC, et al. Prone positioning in severe acute respiratory distress syndrome. N Engl J Med. 2013;368(23):2159-68.

44. Matthay MA, Aldrich JM, Gotts JE. Treatment for severe acute respiratory distress syndrome from COVID-19. Lancet Respir Med. 2020:8(5):433-4. 
45. Vieillard-Baron A, Rabiller A, Chergui K, et al. Prone position improves mechanics and alveolar ventilation in acute respiratory distress syndrome. Intensive Care Med. 2005;31(2):220-6.

46. Jozwiak M, Teboul JL, Anguel N, et al. Beneficial hemodynamic effects of prone positioning in patients with acute respiratory distress syndrome. Am J Respir Crit Care Med. 2013;188(12):1428-33.

47. Fan E, Del Sorbo L, Goligher EC, et al. An Official American Thoracic Society/European Society of Intensive Care Medicine/Society of Critical Care Medicine Clinical Practice Guideline: Mechanical Ventilation in Adult Patients with Acute Respiratory Distress Syndrome [published correction appears in Am J Respir Crit Care Med. 2017 Jun 1;195(11):1540]. Am J Respir Crit Care Med. 2017;195(9):1253-63.

48. Mekontso Dessap A, Proost O, Boissier F, Louis B, Roche Campo F, Brochard L. Transesophageal echocardiography in prone position during severe acute respiratory distress syndrome. Intensive Care Med. 2011;37(3):430-4

49. Ramanathan K, Antognini D, Combes A, et al. Planning and provision of ECMO services for severe ARDS during the COVID-19 pandemic and other outbreaks of emerging infectious diseases. Lancet Respir Med. 2020;8(5):518-26.

50. Doufle G, Roscoe A, Billia F, Fan E. Echocardiography for adult patients supported with extracorporeal membrane oxygenation. Crit Care. 2015;19:326.

51. Hoeper MM, Wiesner O, Hadem J, et al. Extracorporeal membrane oxygenation instead of invasive mechanical ventilation in patients with acute respiratory distress syndrome. Intensive Care Med. 2013;39(11):2056-7.

52. Haller M, Zöllner C, Manert W, et al. Thermodilution cardiac output may be incorrect in patients on venovenous extracorporeal lung assist. Am J Respir Crit Care Med. 1995;152(6 Pt 1):1812-7.

53. Sander M, von Heymann C, Foer A, et al. Pulse contour analysis after normothermic cardiopulmonary bypass in cardiac surgery patients. Crit Care. 2005;9(6):R729-34.

54. Chhina AK, Loyd GE, Szymanski TJ, et al. Frequency and analysis of unplanned extubation in coronavirus disease 2019 patients. Crit Care Explor. 2020;2(12):e0291

55. Mayo P, Volpicelli G, Lerolle $\mathrm{N}$, et al. Ultrasonography evaluation during the weaning process: the heart, the diaphragm, the pleura and the lung. Intensive Care Med. 2016;42(7):1107-17.

56. Caille V, Amiel JB, Charron C, et al. Echocardiography: a help in the weaning process. Crit Care. 2010;14(3):R120.

57. Papanikolaou J, Makris D, Saranteas T, et al. New insights into weaning from mechanical ventilation: left ventricular diastolic dysfunction is a key player. Intensive Care Med. 2011;37(12):1976-85.

58. Soummer A, Perbet $\mathrm{S}$, Brisson $\mathrm{H}$, et al. Ultrasound assessment of lung aeration loss during a successful weaning trial predicts postextubation distress*. Crit Care Med. 2012:40(7):2064-72.

59. Bouhemad B, Mongodi S, Via G, Rouquette I. Ultrasound for "lung monitoring" of ventilated patients. Anesthesiology. 2015;122(2):437-47.

60. Richards-Belle A, Orzechowska I, Gould DW, et al. ICNARC COVID-19 Team. COVID-19 in critical care: epidemiology of the first epidemic wave across England, Wales and Northern Ireland. Intensive Care Med. 2020;46(11):2035-47.

61. Martin-Villares C, Perez-Molina-Ramirez C, Bartolome-Benito M, et al. COVID ORL ESP Collaborative Group. Outcome of 1890 tracheostomies for critical COVID-19 patients: a national cohort study in Spain. Eur Arch Otorhinolaryngol. 2021;278(5):1605-12.

62. Avilés-Jurado FX, Prieto-Alhambra D, González-Sánchez N, et al. Timing, complications, and safety of tracheotomy in critically III patients with COVID-19. JAMA Otolaryngol Head Neck Surg. 2020;147(1):1-8.

63. Angel LF, Amoroso NE, Rafeg S, et al. Percutaneous dilational tracheostomy for coronavirus disease 2019 patients requiring mechanical ventilation. Crit Care Med. 2021:49(7):1058-67.

64. Mehta AB, Syeda SN, Bajpayee L, et al. Trends in tracheostomy for mechanically ventilated patients in the United States, 1993-2012. Am J Respir Crit Care Med. 2015;192:446-54.

65. Queen Elizabeth Hospital Birmingham COVID-19 Airway Team. Safety and 30-day outcomes of tracheostomy for COVID-19: a prospective observational cohort study. Br J Anaesth. 2020;125:872-9.

66. Singh M, Chin KJ, Chan VW, et al. Use of sonography for airway assessment: an observational study. J Ultrasound Med. 2010;29:79-85.
67. Alansari M, Alotair H, Al Aseri Z, et al. Use of ultrasound guidance to improve the safety of percutaneous dilatational tracheostomy: a literature review. Crit Care. 2015:19(1):229.

68. Lhéritier G, Legras A, Caille A, et al. Prevalence and prognostic value of acute cor pulmonale and patent foramen ovale in ventilated patients with early acute respiratory distress syndrome: a multicenter study. Intensive Care Med. 2013;39(10):1734-42.

69. Mekontso Dessap A, Boissier F, et al. Acute cor pulmonale during protective ventilation for acute respiratory distress syndrome: prevalence, predictors, and clinical impact. Intensive Care Med. 2016;42(5):862-70.

70. Vignon P, Merz TM, Vieillard-Baron A. Ten reasons for performing hemodynamic monitoring using transesophageal echocardiography. Intensive Care Med. 2017;43(7):1048-51.

71. Creel-Bulos C, Hockstein M, Amin N, Melhem S, Truong A, Sharifpour M. Acute Cor Pulmonale in Critically III Patients with Covid-19. N Engl J Med. 2020;382(21):e70

72. Tavazzi G, Civardi L, Caneva L, Mongodi S, Mojoli F. Thrombotic events in SARS-CoV-2 patients: an urgent call for ultrasound screening. Intensive Care Med. 2020;46(6):1121-3.

73. McLean AS. Echocardiography in shock management. Crit Care. 2016;20:275.

74. Wang D, Hu B, Hu C, et al. Clinical characteristics of 138 hospitalized patients with 2019 novel coronavirus-infected pneumonia in Wuhan, China. JAMA. 2020;323(11):1061-9.

75. Jia X, Malhotra A, Saeed M, et al. Risk factors for ARDS in patients receiving mechanical ventilation for $>48$ h. Chest. 2008;133(4):853-61.

76. Zochios V, Jones N. Acute right heart syndrome in the critically ill patient. Heart Lung Vessel. 2014;6(3):157-70.

77. Vignon P, Evrard B, Asfar P, et al. Fluid administration and monitoring in ARDS: which management? Intensive Care Med. 2020;46(12):2252-64.

78. Lichtenstein DA. Point-of-care ultrasound: infection control in the intensive care unit. Crit Care Med. 2007;35(5 Suppl):S262-7.

79. Toupin F, Denault A, Lamarche Y, Deschamps A. Hemodynamic instability and fluid responsiveness. Can J Anesth. 2013;60:1240-7.

80. McNaughton DA, Abu-Yousef MM. Doppler US of the liver made simple. Radiographics. 2011;31:161-88.

81. Centers for Disease Control and Prevention. Interim clinical guidance for management of patients with confirmed coronavirus disease (COVID-19). Updated Februrary 12, 2021. https://www.cdc.gov/coronavirus/2019ncov/hcp/clinical-guidance-management-patients.html.

\section{Publisher's Note}

Springer Nature remains neutral with regard to jurisdictional claims in published maps and institutional affiliations.

Ready to submit your research? Choose BMC and benefit from

- fast, convenient online submission

- thorough peer review by experienced researchers in your field

- rapid publication on acceptance

- support for research data, including large and complex data types

- gold Open Access which fosters wider collaboration and increased citations

- maximum visibility for your research: over 100M website views per year

At BMC, research is always in progress.

Learn more biomedcentral.com/submissions 